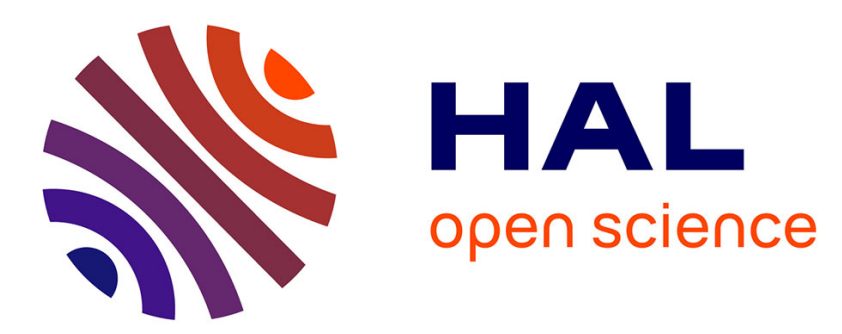

\title{
Inferring Sequences Produced by a Linear Congruential Generator on Elliptic Curves Using Coppersmith's Methods
}

\author{
Thierry Mefenza
}

\section{- To cite this version:}

Thierry Mefenza. Inferring Sequences Produced by a Linear Congruential Generator on Elliptic Curves Using Coppersmith's Methods. COCOON 2016, Aug 2016, Ho Chi Minh City, Vietnam. pp.12, 10.1007/978-3-319-42634-1_24. hal-01381658

\section{HAL Id: hal-01381658 \\ https://hal.inria.fr/hal-01381658}

Submitted on 14 Oct 2016

HAL is a multi-disciplinary open access archive for the deposit and dissemination of scientific research documents, whether they are published or not. The documents may come from teaching and research institutions in France or abroad, or from public or private research centers.
L'archive ouverte pluridisciplinaire HAL, est destinée au dépôt et à la diffusion de documents scientifiques de niveau recherche, publiés ou non, émanant des établissements d'enseignement et de recherche français ou étrangers, des laboratoires publics ou privés. 


\title{
Inferring Sequences Produced by a Linear Congruential Generator on Elliptic Curves using Coppersmith's Methods
}

\author{
Thierry Mefenza \\ Cascade-DIENS, CNRS, INRIA, PSL, Paris, France, and \\ Department of Mathematics, University of Yaounde 1, Cameroon \\ thierrymefenza@yahoo.fr
}

\begin{abstract}
We analyze the security of the Elliptic Curve Linear Congruential Generator (EC-LCG). We show that this generator is insecure if sufficiently many bits are output at each iteration. In 2007, Gutierrez and Ibeas showed that this generator is insecure given a certain amount of most significant bits of some consecutive values of the sequence. Using the Coppersmith's methods, we are able to improve their security bounds.
\end{abstract}

Keywords. Elliptic Curve Linear Congruential Generator, Lattice reduction, Coppersmith's methods, Elliptic Curves.

\section{Introduction}

In cryptography, a pseudo-random number generator is a deterministic algorithm which takes as input a short random seed and outputs a long sequence which is indistinguishable in polynomial time from a truly random sequence. Pseudo-random numbers have found a number of applications in the literature. For instance they are useful in cryptography for key generation, encryption and signature. In 1994, Hallgren [Hal94] proposed a pseudo-random number generator based on a subgroup of points of an elliptic curve defined over a prime finite field. This generator is known as the Linear Congruential Generator on Elliptic Curves (EC-LCG). Let $E$ be an elliptic curve defined over a prime finite field $\mathbb{F}_{p}$, that is a rational curve given by the following Weierstrass equation

$$
E: y^{2}=x^{3}+a x+b
$$

for some $a, b \in \mathbb{F}_{p}$ with $4 a^{3}+27 b^{2} \neq 0$. It is well known that the set $E\left(\mathbb{F}_{p}\right)$ of $\mathbb{F}_{p}$-rational points (including the special point $O$ at infinity) forms an Abelian group with an appropriate composition rule (denoted $\oplus$ ) where $O$ is the neutral element. For a given point $G \in E\left(\mathbb{F}_{p}\right)$, the EC-LCG is a sequence $U_{n}$ of points defined by the relation:

$$
U_{n}=U_{n-1} \oplus G=n G \oplus U_{0}, \quad n \in \mathbb{N}
$$

where $U_{0} \in E\left(\mathbb{F}_{p}\right)$ is the initial value or seed. We refer to $G$ as the composer of the generator. The EC-LCG provides a very attractive alternative to linear 
and non-linear congruential generators and it has been extensively studied in the literature [Shp05,HS02,GL01,GBS00,MS02,BD02]. In cryptography, we want to use the output of the generator as a stream cipher. One can notice that if two consecutive values $U_{n}, U_{n+1}$ of the generator are revealed, it is easy to find $U_{0}$ and $G$. So, we output only the most significant bits of each coordinate of $U_{n}$, $n \in \mathbb{N}$ in the hope that this makes the resulting output sequence difficult to predict. In this paper, we show that the EC-LCG is insecure if sufficiently many bits are output at each stage. Therefore a secure use of this generator requires to output fewer bits at each iteration and the efficiency of the schemes is thus degraded. Our attacks used the well-known Coppersmith's methods for finding small roots on polynomial equations. These methods have been introduced in 1996 by Coppersmith for polynomial of one or two variables [Cop96a,Cop96b] and have been generalized to many variables. These methods have been used to infer many pseudorandom generators and to cryptanalyze many schemes in cryptography (see [BCTV16,BVZ12] and the references therein). In this paper we used such techniques to improve the previous bounds known on the security of the EC-LCG in the literature. Our improvements are theoretical since in practice, the performance of Coppersmith's method in our case is bad because of large dimension of the lattice.

Prior work. In the cryptography setting, the initial value $U_{0}$ and the constants $G, a$ and $b$ may be kept secret. Gutierrez and Ibeas [GI07] consider two cases: the case where the composer $G$ is known and $a, b$ are kept secret and the case where the composer $G$ is unknown and $a, b$ are kept secret. In the first case, they showed that the EC-LCG is insecure if a proportion of at most $1 / 6$ of the least significant bits of two consecutive values of the sequence is hidden. When the composer is unknown, they showed heuristically that the EC-LCG is insecure if a proportion of at most 1/46 of the least significant bits of three consecutive values of the sequence is hidden. Their result is based on a lattice basis reduction attack, using a certain linearization technique. In some sense, their technique can be seen as a special case of the problem of finding small solutions of multivariate polynomial congruences. The Coppersmith's methods also tackle the problem of finding small solutions of multivariate polynomial congruences. Gutierrez and Ibeas due to the special structure of the polynomials involved claimed that "the Coppersmith's methods does not seem to provide any advantages", and that "It may be very hard to give any precise rigorous or even convincing heuristic analysis of this approach". Our purpose in this paper is to tackle this issue.

Our contributions. We infer the EC-LCG sequence using Coppersmith's method for calculating the small roots of multivariate polynomials modulo an integer. The method for multivariate polynomials is heuristic since it is not proven and may fail (but in practice it works most of the time). At the end of the Coppersmith's methods we use the methods from [BCTV16] to analyze the success condition. In the case where the composer is known, we showed that the ECLCG is insecure if a proportion of at most $1 / 5$ of the least significant bits of two consecutive values $U_{0}$ and $U_{1}$ of the sequence is hidden. This improves the previous bound $1 / 6$ of Gutierrez and Ibeas. We further improve this result by 
considering several consecutive values of the sequence. We showed that the ECLCG is insecure if a proportion of at most $3 / 11$ of the least significant bits of these values is hidden. In the case where the composer is unknown, we showed that the EC-LCG is insecure if a proportion of at most 1/24 of the least significant bits of two consecutive values $U_{0}$ and $U_{1}$ of the sequence is hidden. This improves the previous bound $1 / 46$ of Gutierrez and Ibeas. We further improve this result by considering sufficiently many consecutive values of the sequence. We showed that the EC-LCG is insecure if a proportion of at most 1/8 of the least significant bits of these values is hidden.

The table below gives a comparison between our results and those of Gutierrez and Ibeas. It gives the bound of the proportion of least significant bits hidden from each consecutive values necessary to break the EC-LCG in (heuristic) polynomial time. The basic proportion corresponds to the case where the adversary knows bits coming from the minimum number of intermediate values leading to a feasible attack; while the asymptotic proportion corresponds to the case when the bits known by the adversary knows bits coming from arbitrary number of values.

\begin{tabular}{|c|c|c|c|c|}
\hline & \multicolumn{2}{|c|}{ Basic proportion } & \multicolumn{2}{c|}{ Asymptotic proportion } \\
\hline & Prior result & Our result & Prior result & Our result \\
\hline known composer & $1 / 6$ & $1 / 5$ & None & $3 / 11$ \\
\hline unknown composer & $1 / 46$ & $1 / 24$ & None & $1 / 8$ \\
\hline
\end{tabular}

\section{Preliminaries}

For some $\Delta>0$, we say that $W=\left(x_{W}, y_{W}\right) \in \mathbb{F}_{p}^{2}$ is a $\Delta$-approximation to $U=\left(x_{U}, y_{U}\right) \in \mathbb{F}_{p}^{2}$ if there exists integers $e, f$ satisfying:

$$
|e|,|f| \leqslant \Delta, \quad x_{W}+e=x_{U}, \quad y_{W}+e=y_{U} .
$$

Throughout the paper, $\Delta<p^{\delta}$, with $0<\delta<1$, corresponds to the situation where a proportion of at most $\delta$ of the least significant bits of the output sequence remain hidden.

\subsection{The group law on elliptic curves}

In this subsection, we recall the group law $\oplus$ on elliptic curves defined by the Weierstrass equation (for more details on elliptic curves, see [BSS99,Was08]), since our pseudorandom generator is defined recursively by adding a fixed composer $G$ to the previous value. Let $E / \mathbb{F}_{p}: y^{2}=x^{3}+a x+b$ be an elliptic curve over $\mathbb{F}_{p}$. For two points $P=\left(x_{P}, y_{P}\right)$ and $Q=\left(x_{Q}, y_{Q}\right)$, with $P, Q \neq O$ the addition law $\oplus$ is defined as follows:

$$
P \oplus Q=R=\left(x_{R}, y_{R}\right),
$$


- If $x_{P} \neq x_{Q}$, then

$$
x_{R}=m^{2}-x_{P}-x_{Q}, \quad y_{R}=m\left(x_{P}-x_{R}\right)-y_{P}, \quad \text { where, } m=\frac{y_{Q}-y_{P}}{x_{Q}-x_{P}}
$$

- If $x_{P}=x_{Q}$ but $\left(y_{P} \neq y_{Q}\right.$ or $\left.y_{P}=y_{Q}=0\right)$, then $R=O$

- If $P=Q$ and $y_{P} \neq 0$, then

$$
x_{R}=m^{2}-2 x_{P}, \quad y_{R}=m\left(x_{P}-x_{R}\right)-y_{P}, \quad \text { where, } m=\frac{3 x_{Q}^{2}+a}{2 y_{P}}
$$

\subsection{Coppersmith's methods}

In this section, we give a short description of Coppersmith's method for solving a multivariate modular polynomial system of equations modulo an integer $N$. We refer the reader to [JM06] for details and proofs.

Problem definition. Let $f_{1}\left(y_{1}, \ldots, y_{n}\right), \ldots, f_{s}\left(y_{1}, \ldots, y_{n}\right)$ be irreducible multivariate polynomials defined over $\mathbb{Z}$, having a root $\left(x_{1}, \ldots, x_{n}\right)$ modulo a known integer $N$, namely $f_{i}\left(x_{1}, \ldots, x_{n}\right) \equiv 0 \bmod N$. We want this root to be small in the sense that each of its components is bounded by a known value $X_{i}$.

Polynomials collection. In a first step, one generates a collection $\mathfrak{P}$ of polynomials $\left\{\tilde{f}_{1}, \ldots, \tilde{f}_{r}\right\}$ linearly independent having $\left(x_{1}, \ldots, x_{n}\right)$ as a root modulo powers of $N$. Usually, multiples and powers of products of $f_{i}, i \in\{1, \ldots, s\}$ are chosen , namely $\tilde{f}_{\ell}=y_{1}^{\alpha_{1, \ell}} \cdots y_{n}^{\alpha_{n, \ell}} f_{1}^{k_{1, \ell}} \cdots f_{s}^{k_{s, \ell}}$ for some integers $\alpha_{1, \ell}, \ldots, \alpha_{n, \ell}$, $k_{1, \ell}, \ldots, k_{s, \ell}$ for $\ell \in\{1, \ldots, r\}$. Such polynomials satisfy the relation

$\tilde{f}_{\ell}\left(x_{1}, \ldots, x_{n}\right) \equiv 0 \bmod N^{\sum_{i=1}^{s} k_{i, \ell}}$, i.e., there exists an integer $c_{i}$ such that $\tilde{f}_{l}\left(x_{1}, \ldots, x_{n}\right)=c_{i} N^{k_{\ell}}, k_{\ell}=\sum_{j=1}^{s} k_{j, \ell}$.

Monomials. We denote $\mathfrak{M}$ the set of monomials appearing in collection of polynomials $\mathfrak{P}$. Then each polynomial $\tilde{f}_{i}$ can be expressed as a vector with respect to a chosen order on $\mathfrak{M}$. We construct a matrix $\mathcal{M}$ and we define $\mathcal{L}$ the lattice generated by its rows. From that point, one computes an LLL-reduction on the lattice $\mathcal{L}$ and computes the Gram-Schmidt's orthogonalized basis of the LLL output basis. Extracting the coefficients appearing in the obtained vectors, one can construct polynomials defined over $\mathbb{Z}$ such that $\left\{p_{1}\left(x_{1}, \ldots, x_{n}\right)=\right.$ $\left.0, \ldots, p_{n}\left(x_{1}, \ldots, x_{n}\right)=0\right\}$. Under the (heuristic) assumption that all created polynomials define an algebraic variety of dimension 0 , the previous system can be solved (e.g., using elimination techniques such as Groebner basis) and the desired root recovered in polynomial time.

The conditions on the bounds $X_{i}$ that make this method work are given by the following (simplified) inequation (see [JM06] for details):

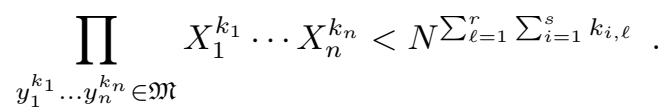


For such techniques, the most complicated part is the choice of the collection of polynomials, what could be a really intricate task when working with multiple polynomials.

\subsection{Analytic Combinatorics}

In the following, we recall the analytic combinatorics methods [FS09] to count the exponents of the bounds $X_{1}, \ldots, X_{n}$ and of the modulo $N$ on the monomials and polynomials appearing in the inequality (2) in Coppersmith's methods. Those methods can be used to compute the cardinalities of the sets $\mathfrak{P}$ and $\mathfrak{M}$. We used the same notations as in [BCTV16] and for more details of the methods the reader is referred to that paper. We see $\mathfrak{P}$ (respectively $\mathfrak{M}$ ) as a combinatorial class with size function $S\left(\tilde{f}_{\ell}\right)=\operatorname{deg}\left(\tilde{f}_{\ell}\right)$ (respectively $S\left(y_{\mathbf{k}}\right)=\operatorname{deg}\left(y_{\mathbf{k}}\right)$, where $\left.y_{\mathbf{k}} \in \mathfrak{M}\right)$. We recall that a combinatorial class is a finite or countable set on which a size function is defined, satisfying the following conditions: (i) the size of an element is a non-negative integer and (ii) the number of elements of any given size is finite. We define another function $\chi$, called a parameter function, such that $\chi\left(\tilde{f}_{\ell}\right)=k_{\ell}$ (respectively $\chi\left(y_{\mathbf{k}}\right)=k_{i}$, where $k_{i}$ is the degree of the variable $y_{i}$ in $\left.y_{\mathbf{k}}\right)$. This allows us to compute for some non negative integer $t, \psi$ (respectively $\alpha_{i}$ ) as:

$$
\psi=\chi_{<t}(\mathfrak{P})=\sum_{a \in \mathfrak{P}: S(a)<t} \chi(a) \quad \alpha_{i}=\chi_{<t}(\mathfrak{M})=\sum_{a \in \mathfrak{P}: S(a)<t} \chi(a) .
$$

To do so we should be able to compute given a combinatorial class $\mathfrak{A}(\mathfrak{A}=\mathfrak{P}$ or $\mathfrak{A}=\mathfrak{M}$ ) with size function $S$ and the parameter function $\chi$,

$$
\chi_{\leqslant p}(\mathfrak{A})=\sum_{a \in \mathfrak{A}: S(a) \leqslant p} \chi(a) .
$$

We proceed as follows:

1. We give another description of $\mathfrak{A}$ with respect to $S$ and $\chi$. This description associates to the combinatorial class an ordinary generating function (OGF) $F(z, u)$ (using Table 1, see [BCTV16] for details). When the class contains elements of different sizes (such as variables of degree 1 and polynomials of degree $e$ ), the variables in the OGF are represented by the atomic element $\mathcal{Z}$ and the polynomials by the element $\mathcal{Z}^{e}$, in order to take into account the degree of these polynomials. Then we "mark" the element useful for the parameter, with a new variable $u$. At this level we only know how to compute $\sum_{a \in \mathfrak{A}: S(a)=p} \chi(a)$. An easier way to compute $\chi_{\leqslant p}(\mathfrak{A})$ is to force all elements $a$ of size less than or equal to $p$ to be of size exactly $p$ by adding enough times a dummy element $y_{0}$ such that $\chi\left(y_{0}\right)=0$. In our context of polynomials, the aim of the dummy variable $y_{0}$ is to homogenize the polynomial.

2. We have:

$$
\chi_{\leqslant}(\mathfrak{A})(z)=\sum_{p=0}^{+\infty} \chi_{\leqslant p}(\mathfrak{A}) z^{p}=\left.\frac{\partial F(z, u)}{\partial u}\right|_{u=1}
$$


Table 1. Combinatorics constructions and their OGF

\begin{tabular}{lll}
\hline & Construction & OGF \\
\hline Atomic class & $\mathcal{Z}$ & $Z(z)=z$ \\
Neutral class & $\varepsilon$ & $E(z)=1$ \\
\hline Disjoint union & $\mathcal{A}=\mathcal{B}+\mathcal{C} \quad($ when $\mathcal{B} \cap \mathcal{C}=\emptyset)$ & $A(z)=B(z)+C(z)$ \\
Complement & $\mathcal{A}=\mathcal{B} \backslash \mathcal{C} \quad($ when $\mathcal{C} \subseteq \mathcal{B})$ & $A(z)=B(z)-C(z)$ \\
Cartesian product & $\mathcal{A}=\mathcal{B} \times \mathcal{C}$ & $A(z)=B(z) \cdot C(z)$ \\
Cartesian exponentiation & $\mathcal{A}=\mathcal{B}^{k}=\mathcal{B} \times \cdots \times \mathcal{B}$ & $A(z)=B(z)^{k}$ \\
Sequence & $\mathcal{A}=\operatorname{SEQ}(\mathcal{B})=\varepsilon+\mathcal{B}+\mathcal{B}^{2}+\ldots$ & $A(z)=\frac{1}{1-B(z)}$ \\
\hline
\end{tabular}

3. Since Coppersmith's method is usually used in an asymptotic way, singularity analysis enables us to find the asymptotic value of the coefficients in an simple way by using the following theorem (see [FS09], page 392):

Theorem 1 (Transfer Theorem). Let $\mathfrak{A}$ be a combinatorial class with an ordinary generating function $F$ regular enough such that there exists a value c verifying

$$
F(z)=\sum_{n=0}^{+\infty} F_{n} z^{n} \underset{z \rightarrow 1}{\sim} \frac{c}{(1-z)^{\alpha}}
$$

for a non-negative integer $\alpha$. The asymptotic value of the coefficient $F_{n}$ is

$$
F_{n} \underset{n \rightarrow \infty}{\sim}\left(c n^{\alpha-1}\right) /(\alpha-1) ! .
$$

\section{Predicting EC-LCG Sequences for Known Composer}

In the cryptographic setting, the initial value $U_{0}=\left(x_{0}, y_{0}\right)$ and the constants $G$, $a$ and $b$ are supposed to be the secret key. In the following, we infer the EC-LCG sequence in the case where the composer $G$ is known and the curve parameters are kept secret. We show that the generator is insecure if at least a proportion of $4 / 5$ of the most significant bits of two consecutive values $U_{0}$ and $U_{1}$ of the sequence is output.

Theorem 2. (two consecutive outputs) Given $\Delta$-approximations $W_{0}, W_{1}$ to two consecutive affine value $U_{0}, U_{1}$ produced by the $E C-L C G$, and given the value of the composer $G=\left(x_{G}, y_{G}\right)$. Under the heuristic assumption that all created polynomials we get by applying Coppersmiths method with the polynomial set $\mathfrak{P}$ below define an algebraic variety of dimension 0 , one can recover the seed $U_{0}$ in heuristic polynomial time in $\log p$ as soon as $\Delta<p^{\delta}$, with $\delta<1 / 5$.

Proof. We suppose without loss of generality that $U_{0} \notin\{-G, G\}$. Then, clearing denominators in (1), we can translate

$$
U_{1}=U_{0} \oplus G
$$

into the following identities in the field $\mathbb{F}_{p}$ :

$$
L_{1}=L_{1}\left(x_{0}, y_{0}, x_{1}\right)=0 \bmod p, \quad L_{2}=L_{2}\left(x_{0}, y_{0}, x_{1}, y_{1}\right)=0 \bmod p
$$


where $U_{0}=\left(x_{0}, y_{0}\right), U_{1}=\left(x_{1}, y_{1}\right)$ and

$$
\begin{gathered}
L_{1}=x_{G}^{3}+x_{1} x_{G}^{2}-x_{0} x_{G}^{2}-2 x_{1} x_{G} x_{0}-x_{G} x_{0}^{2}+x_{0}^{3}+2 y_{G} y_{0}+x_{1} x_{0}^{2}-y_{G}^{2}-y_{0}^{2}, \\
L_{2}=y_{1} x_{G}-y_{1} x_{0}-y_{G} x_{0}+y_{G} x_{1}-y_{0} x_{1}+y_{0} x_{G} .
\end{gathered}
$$

We denote $W_{0}=\left(\alpha_{0}, \beta_{0}\right)$ and $W_{1}=\left(\alpha_{1}, \beta_{1}\right)$. Then using the equalities $x_{j}=\alpha_{j}+e_{j}$ and $y_{j}=\beta_{j}+f_{j}$, for $j \in\{0,1\}$, where $\left|e_{j}\right|,\left|f_{j}\right|<\Delta$ leads to the following polynomial system:

$$
\left\{\begin{aligned}
f\left(e_{0}, e_{1}, f_{0}\right) & =0 \bmod p \\
g\left(e_{0}, e_{1}, f_{0}, f_{1}\right) & =0 \bmod p .
\end{aligned}\right.
$$

where $f\left(z_{1}, z_{2}, z_{3}\right)=A_{1} z_{1}+A_{2} z_{2}+A_{3} z_{3}+A_{4} z_{1}^{2}+A_{5} z_{1} z_{2}+z_{1}^{3}+z_{1}^{2} z_{2}-z_{3}^{2}+A_{6}$ and $g\left(z_{1}, z_{2}, z_{3}, z_{4}\right)=B_{1} z_{1}+B_{2} z_{2}+B_{3} z_{3}+B_{4} z_{4}+z_{1} z_{4}+z_{2} z_{3}+B_{5}$ are polynomials whose coefficients $A_{i}$ 's and $B_{i}$ 's are functions of $x_{G}$, and the approximations values $\alpha_{0}, \alpha_{1}, \beta_{0}, \beta_{1}$. If we set $u_{1}=z_{1}^{3}+z_{1}^{2} z_{2}-z_{3}^{2}$ and $v_{1}=z_{1} z_{4}+z_{2} z_{3}$, then the polynomial $f$ becomes $f_{1}\left(z_{1}, z_{2}, z_{3}, u_{1}\right)=A_{1} z_{1}+A_{2} z_{2}+A_{3} z_{3}+A_{4} z_{1}^{2}+A_{5} z_{1} z_{2}+$ $u_{1}+A_{6}$ and $g$ becomes $g_{1}\left(z_{1}, z_{2}, z_{3}, z_{4}, v_{1}\right)=B_{1} z_{1}+B_{2} z_{2}+B_{3} z_{3}+B_{4} z_{4}+v_{1}+B_{5}$.

Description of the attack. The adversary is therefore looking for the small solutions of the following modular multivariate polynomial system:

$$
\left\{\begin{array}{rl}
f_{1}\left(z_{1}, z_{2}, z_{3}, u_{1}\right) & =0 \bmod p \\
g_{1}\left(z_{1}, z_{2}, z_{3}, z_{4}, v_{1}\right) & =0 \bmod p
\end{array} .\right.
$$

With $\left|z_{j}\right|<\Delta,\left|u_{1}\right|<X=\Delta^{3}$ and $\left|v_{1}\right|<Y=\Delta^{2}$. The attack consists in applying Coppersmith's methods for multivariate polynomials. From now, we use the following collection of polynomials (parameterized by some integer $t \in \mathbb{N})$ :

$\mathfrak{P}=\left\{z_{1}^{j_{1}} \ldots z_{4}^{j_{4}} f_{1}^{i_{1}} g_{1}^{i_{2}} \bmod p^{i_{1}+i_{2}}: i_{1}+i_{2}>0\right.$ and $\left.j_{1}+\cdots+j_{4}+2 i_{1}+i_{2}<2 t\right\}$

The list of monomials appearing within this collection can be described as:

$\mathfrak{M}=\left\{z_{1}^{i_{1}} z_{2}^{i_{2}} z_{3}^{i_{3}} z_{4}^{i_{4}} u_{1}^{i_{5}} v_{1}^{i_{6}} \bmod \Delta^{i_{1}+i_{2}+i_{3}+i_{4}} X^{i_{5}} Y^{i_{6}}: i_{1}+\cdots+i_{4}+2 i_{5}+i_{6}<2 t\right\}$.

If we use for instance the lexicography order on monomials, (with $z_{1}<z_{2}<z_{3}<$ $z_{4}<u_{1}<v_{1}$ ) on the set of monomials, then the leading monomial (denoted $L M$ ) of $f_{1}$ is $\operatorname{LM}\left(f_{1}\right)=u_{1}$ and $\operatorname{LM}\left(g_{1}\right)=v_{1}$. Then the polynomials in $\mathfrak{P}$ are linearly independent since we have prohibited the multiplication by $u_{1}$ and $v_{1}$.

Bounds for the Polynomials modulo $p$. We consider the set $\mathfrak{P}$ as a combinatorial class, with the size function $S\left(z_{1}^{j_{1}} \ldots z_{4}^{j_{4}} f_{1}^{i_{1}} g_{1}^{i_{2}}\right)=j_{1}+\cdots+j_{4}+2 i_{1}+i_{2}$ and the parameter function $\chi\left(z_{1}^{j_{1}} \ldots z_{4}^{j_{4}} f_{1}^{i_{1}} g_{1}^{i_{2}}\right)=i_{1}+i_{2}$. The degree of each variable $z_{i}, u_{1}, v_{1}$ is 1 , whereas the degree of $f_{1}$ is 2 and the degree of $g_{1}$ is 1 . For 
the sake of simplicity, we can consider $0 \leqslant i_{1}+i_{2}$, since the parameter function equals 0 for elements $z_{1}^{j_{1}} \ldots z_{4}^{j_{4}} f_{1}^{i_{1}} g_{1}^{i_{2}}$ with $i_{1}+i_{2}=0$.

We can described $\mathfrak{P}$ as: $\prod_{i=1}^{4} \operatorname{SEQ}(Z) \times \operatorname{SEQ}\left(u Z^{2}\right) \times \operatorname{SEQ}(u Z) \times \operatorname{SEQ}(Z)$, where the last term is for the dummy value $z_{0}$.

This leads to the generating function:

$$
F(z, u)=\left(\frac{1}{1-z}\right)^{5} \times \frac{1}{1-u z^{2}} \times \frac{1}{1-u z} .
$$

As $z \rightarrow 1,1-z^{n} \sim n(1-z)$ leads to:

$$
\left.\frac{\partial F}{\partial u}(u, z)\right|_{u=1} \underset{z \rightarrow 1}{\sim} \frac{3(1-z)}{4(1-z)^{9}} \sim \frac{3}{4(1-z)^{8}},
$$

since $2 t \sim 2 t-1$, this leads to: $\chi<2 t(\mathfrak{P}) \sim \frac{3}{4} \times \frac{(2 t)^{7}}{7 !}$

Bounds for the monomials modulo $\Delta$. We consider the set $\mathfrak{M}$ as a combinatorial class, with the size function $S\left(z_{1}^{i_{1}} \ldots z_{4}^{i_{4}} u^{i_{5}} v^{i_{6}}\right)=i_{1}+\cdots+i_{4}+2 i_{5}+i_{6}$ and the parameter function $\chi\left(z_{1}^{i_{1}} \ldots z_{4}^{i_{4}} u^{i_{5}} v^{i_{6}}\right)=i_{1}+\cdots+i_{4}$. As $z_{1}, z_{2}, z_{3}, z_{4}, u, v$ "count for" $1,1,1,1,2$ and 1 respectively in the condition of the set, we can described $\mathfrak{M}$ as: $\operatorname{SEQ}\left(Z^{2}\right) \times \operatorname{SEQ}(Z) \times \prod_{i=1}^{4} \operatorname{SEQ}(u Z) \times \operatorname{SEQ}(Z)$, where the last term is for the dummy value $z_{0}$.

Which leads to the generating function: $F(z, u)=\frac{1}{\left(1-z^{2}\right)(1-z)^{2}} \times\left(\frac{1}{1-u z}\right)^{4}$. As previously, we obtain $\chi<2 t, \Delta(\mathfrak{M}) \sim \frac{2(2 t)^{7}}{7 !}$.

Bounds for the monomials modulo $X$ (respectively modulo $Y$ ). We consider the set $\mathfrak{M}$ as a combinatorial class, with the size function $S\left(z_{1}^{i_{1}} \ldots z_{4}^{i_{4}} u^{i_{5}} v^{i_{6}}\right)=$ $i_{1}+\cdots+i_{4}+2 i_{5}+i_{6}$ and the parameter function $\chi\left(z_{1}^{i_{1}} \ldots z_{4}^{i_{4}} u^{i_{5}} v^{i_{6}}\right)=i_{5}$ (respectively $\left.\chi\left(z_{1}^{i_{1}} \ldots z_{4}^{i_{4}} u^{i_{5}} v^{i_{6}}\right)=i_{6}\right)$. As $z_{1}, z_{2}, z_{3}, z_{4}, u, v$ "count for" $1,1,1,1,2$ and 1 respectively in the condition of the set, we can described $\mathfrak{M}$ as: $\prod_{i=1}^{5} \operatorname{SEQ}(Z) \times$ $\operatorname{Seq}\left(u Z^{2}\right) \times \operatorname{Seq}(Z)\left(\right.$ respectively $\left.\prod_{i=1}^{4} \operatorname{Seq}(Z) \times \operatorname{Seq}\left(Z^{2}\right) \times \operatorname{Seq}(u Z) \times \operatorname{SeQ}(Z)\right)$ where the last one is for the dummy value $z_{0}$.

Which leads to the generating function: $F(z, u)=\frac{1}{(1-z)^{6}} \times \frac{1}{1-u z^{2}}$ (respectively $\left.F(z, u)=\frac{1}{(1-z)^{5}\left(1-z^{2}\right)} \times \frac{1}{1-u z}\right)$. This leads to: $\chi_{<2 t, X}(\mathfrak{M}) \sim \frac{(2 t)^{7}}{4 \times 7 !}$ (respectively $\left.\chi_{<2 t, Y}(\mathfrak{M}) \sim \frac{(2 t)^{7}}{2 \times 7 !}\right)$.

Condition. We denote by $\nu_{1}=\chi_{<2 t, \Delta}(\mathfrak{M}), \nu_{2}=\chi_{<2 t, X}(\mathfrak{M}), \nu_{3}=\chi_{<2 t, Y}(\mathfrak{M})$ and $\varepsilon=\chi_{<2 t}(\mathfrak{P})$. The inequality $(2)$ is $p^{\varepsilon}>\Delta^{\nu_{1}} X^{\nu_{2}} Y^{\nu_{3}}$, ie $\Delta<p^{\frac{\varepsilon}{\nu_{1}+3 \nu_{2}+2 \nu_{3}}}$, where:

$$
\frac{\varepsilon}{\nu_{1}+3 \nu_{2}+2 \nu_{3}} \sim \frac{\chi_{<2 t}(\mathfrak{P})}{\chi_{<2 t, \Delta}(\mathfrak{M})+3 \chi_{<2 t, X}(\mathfrak{M})+2 \chi_{<2 t, Y}(\mathfrak{M})} \sim \frac{1}{5},
$$

this leads to the claimed bound: $\Delta<p^{\frac{1}{5}}$. 
This bound improves the known bound $\Delta<p^{1 / 6}$. Next we further improve the previous bound and we show that the generator is insecure if at least a proportion of $8 / 11$ of the most significant bits of an arbitrary large number of consecutive values $U_{i}$ of the sequence is output.

Theorem 3. (more consecutive outputs)

Given $\Delta$-approximations $W_{0}, W_{1}, \ldots, W_{n}$ (for some integer $n>1$ ) to $n+1$ consecutive affine values $U_{0}, U_{1}, \ldots, U_{n}$ produced by the $E C-L C G$, and given the value of the composer $G=\left(x_{G}, y_{G}\right)$. Under the heuristic assumption that all created polynomials we get by applying Coppersmiths method with the polynomial set $\mathfrak{P}$ below define an algebraic variety of dimension 0 , one can recover the seed $U_{0}$ in polynomial time in $\log p$ as soon as $\Delta<p^{\delta}$, with $\delta<\frac{3 n}{11 n+4}$.

Proof. (Sketch) We can generalize the previous proof by considering $n$ couples of consecutive values $\left(U_{i}, U_{i+1}\right), i \in\{0, \ldots, n-1\}$ and the same variable change to get $n$ couple of polynomials $f_{i+1}, g_{i+1}$ of the same shape as $f_{1}$ and $g_{1}$. We then apply the method to the following collection of polynomials:

$$
\mathfrak{P}=\left\{\begin{array}{l}
z_{0}^{j_{0}} \ldots z_{2 n+1}^{j_{2 n+1}} f_{1}^{i_{1}} \ldots f_{n}^{i_{n}} g_{1}^{l_{1}} \ldots g_{n}^{l_{n}} \bmod p^{i_{1}+l_{1} \cdots+i_{n}+l_{n}} \\
\text { s.t. } i_{1}+l_{1}+\cdots+i_{n}+l_{n}>0 \\
\text { and } j_{0}+\cdots+j_{2 n+1}+2\left(i_{1}+\cdots+i_{n}\right)+l_{1}+\cdots+l_{n}<2 t
\end{array}\right\},
$$

and the following set of monomials:

$$
\mathfrak{M}=\left\{\begin{array}{l}
z_{0}^{j_{0}} \ldots z_{2 n+1}^{j_{2 n+1}} u_{1}^{i_{1}} v_{1}^{l_{1}} \ldots u_{n}^{i_{n}} v_{n}^{l_{n}} \bmod \Delta^{j_{0}+\cdots+j_{2 n+1}} X^{i_{0}+\cdots+i_{n}} Y^{l_{0}+\cdots+l_{n}} \\
\text { s.t. } j_{0}+\cdots+j_{2 n+1}+2\left(i_{1}+\cdots+i_{n}\right)+l_{1}+\cdots+l_{n}<2 t
\end{array}\right\}
$$

to get the result (see the full version of the paper for the complete proof).

\section{Predicting EC-LCG Sequences for Unknown Composer}

In this section, we infer the EC-LCG sequence in the case where the composer $G$ is unknown and the curve parameters are kept secret. In the following, We show that the generator is insecure if at least a proportion of $23 / 24$ of the most significant bits of three consecutive values $U_{0}$ and $U_{1}$ and $U_{2}$ of the sequence is output.

Theorem 4. (three consecutive outputs) Given $\Delta$-approximations $W_{0}, W_{1}$, $W_{2}$ to three consecutive affine values $U_{0}, U_{1}, U_{2}$ produced by the EC-LCG. Under the heuristic assumption that all created polynomials we get by applying Coppersmiths method with the polynomial set $\mathfrak{P}$ below define an algebraic variety of dimension 0 , one can recover the seed $U_{0}$ and the composer $G$ in polynomial time in $\log p$ as soon as $\Delta<p^{\delta}$ with $\delta<1 / 24$.

Proof. We set $U_{0}=\left(x_{0}, y_{0}\right), U_{1}=\left(x_{1}, y_{1}\right), U_{2}=\left(x_{2}, y_{2}\right), W_{0}=\left(\alpha_{0}, \beta_{0}\right), W_{1}=$ $\left(\alpha_{1}, \beta_{1}\right)$ and $W_{2}=\left(\alpha_{2}, \beta_{2}\right)$. We then have the equalities:

$$
x_{i}=\alpha_{i}+e_{i}, y_{j}=\beta_{j}+f_{j}, \quad \text { where }\left|e_{i}\right|,\left|f_{i}\right|<\Delta, i \in\{0,1,2\} .
$$


We also have:

$$
\left\{\begin{array}{l}
y_{0}^{2}=x_{0}^{3}+a x_{0}+b \\
y_{1}^{2}=x_{1}^{3}+a x_{1}+b \\
y_{2}^{2}=x_{2}^{3}+a x_{2}+b
\end{array}\right.
$$

Eliminating the curve parameters $a, b$ and assuming without loss of generality that $U_{2} \neq \pm U_{1}$ (that is, $x_{2} \neq x_{1}$ ), we obtain the following equation:

$y_{2}^{2}\left(x_{0}-x_{1}\right)+x_{2}^{3}\left(x_{1}-x_{0}\right)+x_{0}^{3}\left(x_{2}-x_{1}\right)+y_{0}^{3}\left(x_{1}-x_{2}\right)+x_{1}^{3}\left(x_{0}-x_{2}\right)+y_{1}^{2}\left(x_{2}-x_{0}\right)=0$

Using the equalities (3), leads to the equation:

$$
f\left(e_{0}, e_{1}, e_{2}, f_{0}, f_{1}, f_{2}\right)=0 \bmod p
$$

where $f$ is a polynomial of degree 4 whose coefficients are functions of $\alpha_{0}, \alpha_{1}, \alpha_{2}$, $\beta_{0}, \beta_{2}$, and $\beta_{2}$.

Description of the attack. The adversary is therefore looking for the solutions smaller than $\Delta$ of the following modular multivariate polynomial equation:

$$
f\left(z_{1}, \ldots, z_{6}\right)=0 \bmod p
$$

The attack consists in applying Coppersmith's methods as in the former subsection. If we consider monomials with respect to lexicographic order, then the leading monomial of $f$ is $z_{1}^{3} z_{2}$. From now on, we use the following collection of polynomials:

$$
\begin{gathered}
\mathfrak{P}=\left\{\tilde{f}_{j_{1}, \ldots, j_{6}, i}=z_{1}^{j_{1}} \ldots z_{6}^{j_{6}} f^{i} \bmod p^{i}: i>0 \text { and } j_{1}+\cdots+j_{6}+4 i<4 t\right. \\
\text { and } \left.\left(0 \leqslant j_{1}<3 \vee j_{2}=0\right)\right\},
\end{gathered}
$$

One can check that the polynomials $\tilde{f}_{j_{1}, \ldots, j_{6}, i}$ are linearly independent since $L M(f) \neq z_{1}^{j_{1}} \ldots z_{6}^{j_{6}}$ for each $\tilde{f}_{j_{1}, \ldots, j_{6}, i}$ from $\mathfrak{P}$. The list of monomials appearing within this collection can be described as:

$$
\mathfrak{M}=\left\{z_{1}^{j_{1}} \ldots z_{6}^{j_{6}} \bmod \Delta^{j_{1}+\cdots+j_{6}}: j_{1}+\cdots+j_{6}<4 t\right\} .
$$

Bounds for the Polynomials modulo $p$. We consider the set $\mathfrak{P}$ as a combinatorial class, with the size function $S\left(\tilde{f}_{j_{1}, \ldots, j_{6}, i}\right)=j_{1}+\cdots+j_{6}+4 i$ and the parameter function $\chi\left(\tilde{f}_{j_{1}, \ldots, j_{6}, i}\right)=i$. Since the degree of each variable $z_{i}$ is 1 and the degree of $f$ is 4 , we can described $\mathfrak{P}$ as:

$\prod_{i=1}^{4} \operatorname{SEQ}(Z) \times \operatorname{SEQ}\left(u Z^{4}\right) \times((\underbrace{\varepsilon+Z+Z^{2}}_{z_{1}})(\underbrace{\varepsilon+Z \operatorname{SEQ}(Z)}_{z_{2}})+\underbrace{Z^{3} \operatorname{SeQ}(Z)}_{z_{1}}) \times \operatorname{SEQ}(Z)$,

where the last term is for the dummy value $z_{0}$. This leads to the generating function:

$$
F(z, u)=\left(\frac{1}{1-z}\right)^{5} \times \frac{1}{1-u z^{4}} \times\left(\left(1+z+z^{2}\right)(1+z /(1-z))+\frac{z^{3}}{1-z}\right) .
$$

This leads to: $\chi_{<4 t}(\mathfrak{P}) \sim \frac{1}{4} \times \frac{(4 t)^{7}}{7 !}$ 
Bounds for the monomials modulo $\Delta$. We consider the set $\mathfrak{M}$ as a combinatorial class, with the size function $S\left(z_{1}^{j_{1}} \ldots z_{6}^{j_{6}}\right)=j_{1}+\cdots+j_{6}$ and the parameter function $\chi\left(z_{1}^{j_{1}} \ldots z_{6}^{j_{6}}\right)=j_{1}+\cdots+j_{6}$. Since the degree of each $z_{i}$ is 1 , we can then described $\mathfrak{M}$ as: $\prod_{i=1}^{6} \operatorname{SeQ}(u Z) \times \operatorname{SeQ}(Z)$, where the last term is for the dummy value $z_{0}$. Which leads to the generating function: $F(z, u)=\left(\frac{1}{1-u z}\right)^{6} \times \frac{1}{1-z}$. We then obtain: $\chi_{<4 t}(\mathfrak{M}) \sim \frac{6(3 t)^{7}}{7 !}$

Condition. If we denote by $\nu=\chi_{<4 t}(\mathfrak{P})$, and $\varepsilon=\chi_{<4 t}(\mathfrak{M})$, the inequality (2) is $p^{\nu}>\Delta^{\varepsilon}$, ie $\Delta<p^{\frac{\nu}{\varepsilon}}$, where: $\frac{\nu}{\varepsilon} \sim \frac{\chi<4 t(\mathfrak{P})}{\chi_{<4 t}(\mathfrak{M})} \sim \frac{1}{24}$, this leads to the claimed bound: $\Delta<p^{\frac{1}{24}}$.

This bound improves the known bound $\Delta<p^{1 / 46}$. Next, we further improve the previous bound and we show that the generator is insecure if at least a proportion of $7 / 8$ of the most significant bits of an arbitrary large number of consecutive values $U_{i}$ of the sequence is output.

Theorem 5. (more consecutive outputs)

Given $\Delta$-approximations $W_{0}, W_{1}, \ldots, W_{n+1}$ (for some integer $n>1$ ) to $n+2$ consecutive affine values $U_{0}, U_{1}, \ldots, U_{n+1}$ produced by the EC-LCG. Under the heuristic assumption that all created polynomials we get by applying Coppersmiths method with the polynomial set $\mathfrak{P}$ below define an algebraic variety of dimension 0 , one can recover the seed $U_{0}$ and the composer $G$ in polynomial time in $\log p$ as soon as $\Delta<p^{\delta}$ with $\delta<n / 4(2 n+4)$.

Proof. See the full version of the paper.

\section{Conclusion}

We analyzed the security of the Elliptic Curve Linear Congruential Generator (EC-LCG). In the case where the composer is known, we showed that this generator is insecure if at least a proportion of $8 / 11$ of the most significant bits of an arbitrary large number of consecutive values $U_{i}$ of the sequence is output. We also consider the cryptographic setting where the composer is unknown and we showed that this generator is insecure if at least a proportion of $7 / 8$ of the most significant bits of an arbitrary large number of consecutive values $U_{i}$ of the sequence is output. Our results are theoretical since in practice, the performance of Coppersmith's method in our attacks is bad because of large dimension of the constructed lattice but they are good evidences of the weaknesses of this generator. This generator should then be used with great care.

Acknowledgments The author was supported in part by the French ANR JCJC ROMAnTIC project (ANR-12-JS02-0004) and by the Simons foundation Pole PRMAIS. I would like to thank anonymous referees for their helpful comments. 


\section{References}

[BCTV16] F. Benhamouda, C. Chevalier, A. Thillard, and D. Vergnaud. Easing coppersmith methods using analytic combinatorics: Applications to public-key cryptography with weak pseudorandomness. In C. -M. Cheng, et al, editors, PKC 16, PartII, volume 9615 of Lect. Notes Comput. Sci., pages 36-66, 2016.

[BD02] P. Beelen, and J. Doumen. Pseudorandom sequences from elliptic curves. Finite fields with applications to coding theory. Cryptography and related areas.Springer-Verlag, Berlin, pages 37-52, 2002.

[BSS99] I. F. Blake, G. Seroussi, and N. P. Smart. Elliptic curves in cryptography. Cambridge: Cambridge University Press, 1999.

[BVZ12] A. Bauer, D. Vergnaud, and J-C. Zapalowicz. Inferring sequences produced by nonlinear pseudorandom number generators using Coppersmith's methods. In M. Fischlin, J. Buchmann, and M. Manulis, editors, PKC 12, volume 7293 of Lect. Notes Comput. Sci., pages 609-626, 2012.

[Cop96a] D. Coppersmith. Finding a small root of a univariate modular equation. In U. M. Maurer, editor, EUROCRYPT 96, volume 1070 of Lect. Notes Comput. Sci., pages 155-165, 1996.

[Cop96b] D. Coppersmith. Finding a small root of a bivariate integer equation; factoring with high bits known. In U. M. Maurer, editor, EUROCRYPT 96, volume 1070 of Lect. Notes Comput. Sci., pages 178-189, 1996.

[FS09] P. Flajolet and R. Sedgewick. Analytic Combinatorics. Cambridge University Press (January 2009).

[GBS00] G. Gong, T. A. Berson, and D. A. Stinson. Elliptic curve pseudorandom sequence generators. volume 1758 of Lect. Notes Comput. Sci., pages 34-49, 2000.

[GI07] J. Gutierrez and A. Ibeas. Inferring sequences produced by a linear congruential generator on elliptic curves missing high-order bits. Design Code Cryptogr, 45:199-212, 2007.

[GL01] G. Gong and C. C. Y. Lam. Linear recursive sequences over elliptic curves. In: Proc. intern. conf. on sequences and their applications, Bergen 2001. Springer-Verlag, London, pages 182-196, 2001.

[Hal94] S. Hallgren. Linear congruential generators over elliptic curves. Preprint CS-94-143, Dept. of Comp. Sci., 1994.

[HS02] F. Hess and I. E. Shparlinski. On the linear complexity and multidimensional distribution of congruential generators over elliptic curves. Design Code Cryptogr, 35:111-117, 2005.

[JM06] E. Jochemsz, and A. May. A strategy for finding roots of multivariate polynomials with new applications in attacking RSA variants. In X. Lai, K. Chen, editors, ASIACRYPT 06, volume 4284 of Lect. Notes Comput. Sci., pages 267-282, 2006.

[MS02] E. Mahassni and I. E. Shparlinski. On the uniformity of distribution of congruential generators over elliptic curves. In: Proc. intern. conf. on sequences and their applications. Bergen 2001. Springer-Verlag, London , pages 257264, 2002.

[Shp05] I. E. Shparlinski. Pseudorandom points on elliptic curves over finite fields. Preprint, 2005.

[Was08] L. C. Washington. Elliptic curves. Number theory and cryptography. 2nd ed. Boca Raton, FL: Chapman and Hall/CRC, 2nd ed. edition, 2008. 